\section{Erst der Krebs, dann die Gürtelrose}

Von einigen Krebsarten ist bekannt, dass sie mit einem erhöhten Risiko für Herpes Zoster einhergehen. Britische Ärzte haben nun Zusammenhänge zwischen den 21 häufigsten Krebserkrankungen und Herpes Zoster untersucht.

In einer Fall-Kontroll-Studie wurden 192.081 britische Zoster-Patienten mit 732.035 entsprechenden Kontrollpersonen ohne diese Infektion gematcht. Die Rückschau zeigte, dass bei 8,4\% der Studienteilnehmer, die einen Herpes Zoster entwickelten, zuvor eine Krebserkrankung bestanden hatte, aber nur bei $6,7 \%$ der Kontrollpatienten (adjustierte Odds Ratio [OR] 1,29). Im Mittel waren rund sechs Jahre von der Krebsdiagnose bis zum Auftreten der Gürtelrose vergangen. Dabei hing die Höhe des Risikos stark von der Art des Tumors ab. Besonders deutlich wurde der Zusammenhang bei hämatologischen Malignomen (OR 2,46). Myelom-Patienten etwa entwickelten mehr als viermal so häufig eine Gürtelrose. Krebsfreie Kontrollen und auch das Risiko nach Lymphomen oder Leukämi- en war in der adjustierten Analyse mehr als verdoppelt. Signifikant gehäuft zeigte sich ein Herpes Zoster zudem bei Patienten mit Krebserkrankungen des ZNS (adj. OR 2,31), während das Risiko bei Karzinomen von Mundhöhle, Ösophagus, Magen, Kolon und Rektum, Lunge, Brust, Ovarien, Prostata, Nieren und Blase um $10-50 \%$ erhöht war. Der stärkste Zusammenhang ergab sich bis zu drei Jahre nach der Krebsdiagnose. Mit zunehmendem Alter nahm das Risiko ab, sowohl nach hämatologischen als auch nach soliden Malignomen.

Fazit: Bei Patienten mit Krebserkrankungen hat sich ein durchschnittlich um 29 \% erhöhtes Risiko für das Auftreten eines Herpes Zoster gezeigt. Besonders ausgeprägt war der Zusammenhang in den ersten Jahren nach der Tumordiagnose sowie bei jüngeren Patienten. Zwei Mechanismen könnten diesem Zusammenhang zugrunde liegen, so die Autoren. Einerseits könnten beide Erkrankungen auf dem Boden einer Immunschwäche entstehen, andererseits könnte aber auch die maligne Erkrankung selbst und/oder deren Therapie die Dysfunktion des Immunsystems verursachen und damit die Entwicklung des Herpes Zoster begünstigen.

Die Ergebnisse ihrer Studie könnten dazu veranlassen, nach den ersten charakteristischen Zoster-Symptomen bei Krebspatienten möglichst schnell mit einer antiviralen Therapie zu beginnen, so die Autoren. Auch eine Zoster-Impfung, sehr früh nach der Krebsdiagnose und vor Beginn immunsuppressiver Therapien, halten sie für angebracht, sobald deren Sicherheit und Effektivität belegt seien. Dr. Christine Starostzik

Hansson E. et al. Herpes zoster risk after 21 specific cancers: population-based casecontrol study. Br J Cancer 2017; 116: 1643-51

\title{
Dieser Ausschlag stammt von einem Glucagonom
}
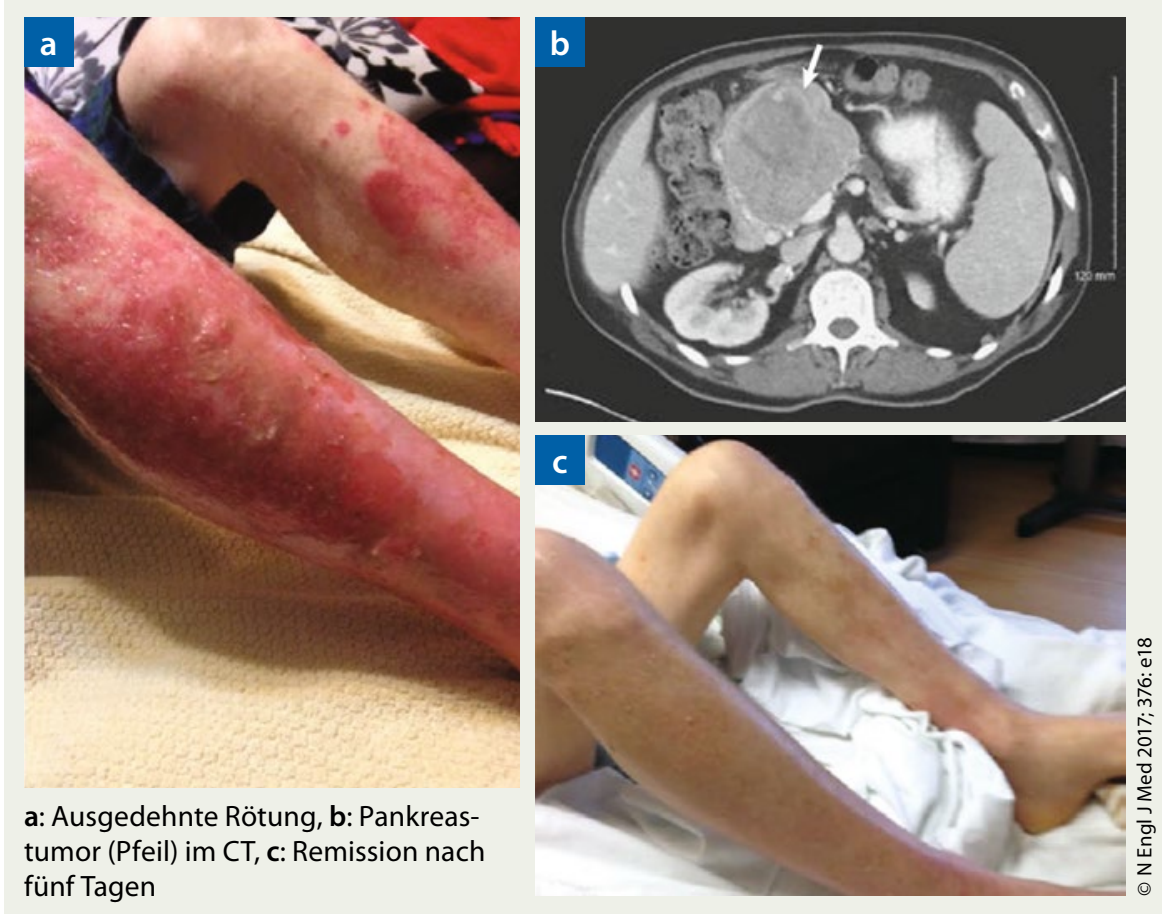

Ein 65-jähriger Mann mit Typ-2-Diabetes hatte innerhalb von drei Monaten 7-8 kg an Gewicht verloren, während sich die Diabeteseinstellung laufend verschlechterte. Der Mann hatte Schmerzen in den Flanken und eine frühes Sättigungsgefühl. Schon vorher hatte sich über drei bis vier Wochen hinweg ein Erythem entwickelt, das sich von den Armen über das Gesäß bis in die Beine ausgebreitet hatte (Abb. a). Der führende Laborbefund war eine Hyperglykämie mit einem Blutzuckerspiegel von 982 mg/dl. Im Abdomen-CT zeigte sich ein $9 \mathrm{~cm}$ großer Tumor im Bereich des Pankreaskopfs (Abb. b). Die Glucagon-Konzentration im Nüchternzustand betrug $530 \mathrm{pg} / \mathrm{ml}$ (Normalwert $\leq 80 \mathrm{pg} / \mathrm{ml}$ ). In einer Operation entfernte man eine glatt begrenzte Raumforderung, die sich histologisch als neuroendokriner Tumor erwies.

Prof. Hermann S. FüeßI

Mountjoy L et al. Glucagonoma-associated rash. N Engl J Med 2017; 376: e18 